\title{
Preliminary Structural Design Considerations and Mass Efficiencies for Lunar Surface Manipulator Concepts
}

\author{
John T. Dorsey* \\ NASA Langley Research Center, Hampton VA, 23681 \\ Martin M. Mikulas ${ }^{\dagger}$ \\ National Institute of Aerospace, Hampton VA, 23681 \\ and \\ William R. Doggett ${ }^{*}$ \\ NASA Langley Research Center, Hampton VA, 23681
}

\begin{abstract}
The mass and sizing characteristics of manipulators for Lunar and Mars planetary surface applications are investigated by analyzing three structural configurations: a simple cantilevered boom with a square tubular cross-section; a hybrid cable/boom configuration with a square tubular cross-section support structure; and a hybrid cable/boom configuration with a square truss cross-section support structure. Design procedures are developed for the three configurations and numerical examples are given. A new set of performance parameters are developed that relate the mass of manipulators and cranes to a loading parameter. These parameters enable the masses of different manipulator configurations to be compared over a wide range of design loads and reach envelopes (radii). The use of these parameters is demonstrated in the form of a structural efficiency chart using the newly considered manipulator configurations. To understand the performance of Lunar and Mars manipulators, the design procedures were exercised on the three manipulator configurations assuming graphite/epoxy materials for the tubes and trusses. It is also assumed that the actuators are electric motor, gear reduction systems. Numerical results for manipulator masses and sizes are presented for a variety of manipulator reach and payload mass capabilities. Results are presented that demonstrate the sensitivity of manipulator mass to operational radius, tip force, and actuator efficiency. The effect of the value of gravitational force on the ratio of manipulator-mass to payload-mass is also shown. Finally, results are presented to demonstrate the relative mass reduction for the use of graphite/epoxy compared to aluminum for the support structure.
\end{abstract}

\section{Nomenclature}

$\mathrm{a}$

$\mathrm{a}_{\operatorname{Tr}}$

b

c

$\mathrm{c}_{\mathrm{b}}$

$\mathrm{d}_{\mathrm{ch}}$

$\mathrm{d}_{\mathrm{cv}}$

$\mathrm{E}_{\mathrm{T}}$

$\mathrm{E}_{\mathrm{T}} \mathrm{r}$

F

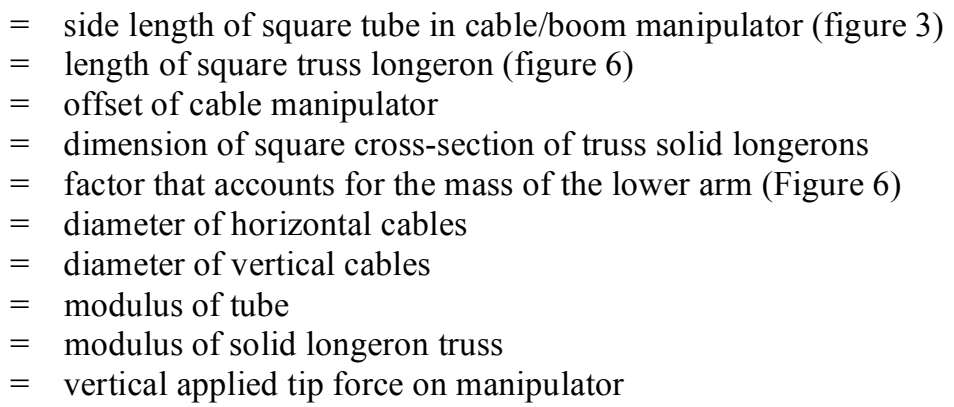

\footnotetext{
* Senior Research Engineer, Structural Concepts and Mechanics Branch, MS-190, Associate Fellow, AIAA.

${ }^{\dagger}$ Senior Research Fellow, Fellow, AIAA.

* Senior Research Engineer, Structural Concepts and Mechanics Branch, MS-190, Member AIAA. 


$\begin{array}{ll}\mathrm{FS}_{\mathrm{c}} & =\text { factor of safety of cable } \\ \mathrm{FS}_{\text {rods }} & =\text { factor of safety of rods } \\ \mathrm{FS}_{\text {Truss }} & =\text { factor of safety of truss } \\ \mathrm{FS}_{\text {Tube }} & =\text { factor of safety of tubes } \\ \mathrm{j} & =\text { factor to account for joint mass } \\ \mathrm{K}_{\mathrm{A}} & =\text { actuator torque/mass constant } \\ \mathrm{K}_{\mathrm{H}} & =\text { hoist torque/mass constant } \\ \mathrm{LWH}_{\mathrm{W}} & =\text { Factor to account for hoist level wind mechanism and drum diameter build-up } \\ \mathrm{M}_{\mathrm{a}} & =\text { mass of actuator } \\ \mathrm{M}_{\mathrm{p}} & =\text { mass of tip payload } \\ \mathrm{Me} & =\text { self loading mass of manipulator due to gravity } \\ \mathrm{n} & =\text { number of cables supporting load } \\ \mathrm{R} & =\text { maximum operating length of manipulator (figure 5) } \\ \mathrm{t} & =\text { assumed thickness of manipulator tubes } \\ \beta & =\text { ratio of truss batten and diagonal area to that of longerons } \\ \gamma & =\text { ratio of mass of the tube, joints, and actuators, to mass of the tubes for merit performance chart } \\ \Delta & =\text { maximum deflection of boom tip } \\ \rho_{\mathrm{C}} & =\text { effective density of cable based on max outside diameter } \\ \rho_{\text {rod }} & =\text { rod density } \\ \rho_{\mathrm{T}} & =\text { tube density } \\ \sigma_{\mathrm{c}} & =\text { ultimate strength of cable } \\ \sigma_{\text {rod }} & =\text { ultimate strength of rods } \\ \sigma_{\mathrm{T}} & =\text { Strength of manipulator materials } \\ \eta & =\text { ratio of king post length to manipulator radius R (figure 5) }\end{array}$

\section{Introduction}

Devices for lifting and transporting payloads and material are critical for efficient Earth-based construction operations. Recent studies have demonstrated that devices with similar functionality will be needed to support lunar outpost operations such as unloading landers (see figure 1), construction, servicing, inspection, payload placement, regolith excavation and grading (see references $1-4)$. Over the years a large number of structural concepts have been conceived and developed for various Earthbased manipulators and cranes as discussed in reference 5. However, devices used on the lunar surface will require a very different design and set of features compared to Earthbased devices. Since the cost to transport mass to the lunar surface is very high, the number of devices that can be dedicated to surface operations will be limited. Thus, in contrast to Earth-based construction, where several dedicated devices can be deployed, lunar missions will require devices that are versatile and multi-purpose. In addition, Earth-based devices generally rely on hydraulic systems to apply and react large

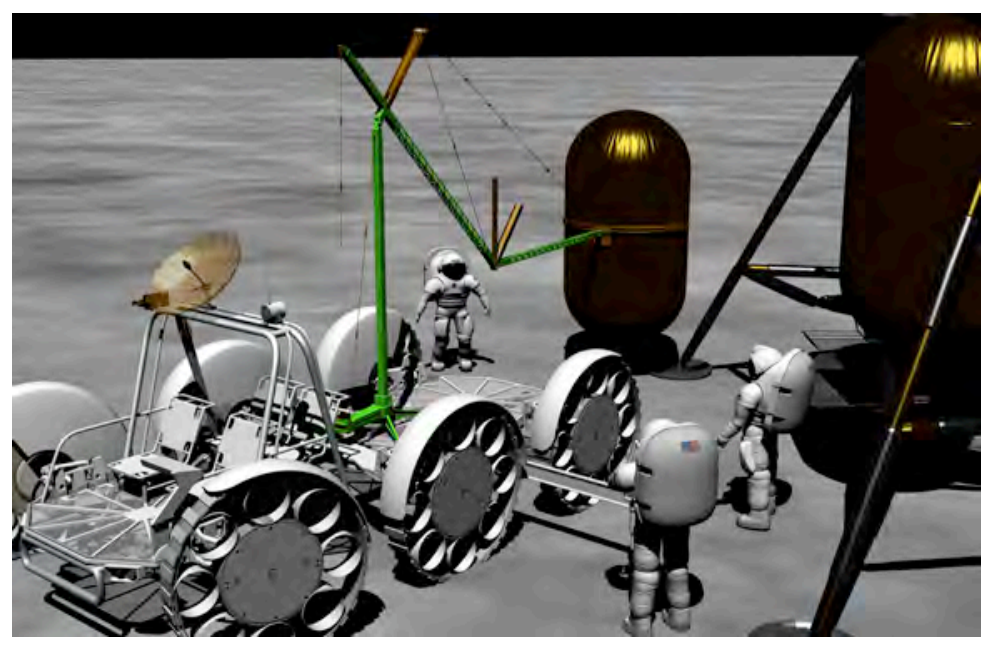

Figure 1. Lunar Surface Manipulation System removing tank from lander. forces and moments, and are constructed from heavy but inexpensive materials such as steel for the device structures. Due to the vacuum environment, lunar devices most likely cannot rely on hydraulic systems. In addition, the devices must package efficiently for launch and be very mass efficient.

The selection of a manipulator structural concept for a given space application is complicated by the large number of requirements that are involved in the evaluation process. For Lunar or planetary applications, low mass is a major design driver. However, the device must also be capable of compact packaging for launch (which imposes additional constraints on member dimensions). In order to identify the major governing parameters for manipulator 
design, a first principles study is performed for a simple cantilevered boom as shown in figure 2-a. Although manipulator sizing can involve numerous design requirements such as strength, stiffness, stability and frequency, the major governing parameters are derived considering only a material strength condition. A set of parameters, in the form of a structural index chart, are subsequently shown to provide a basis for comparing a wide variety of manipulators with different design requirements. In this study, equations that can be used to size the structural members and estimate the structural mass

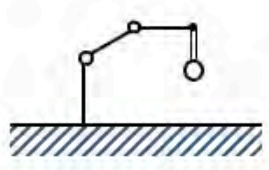

a. Boom Manipulator.

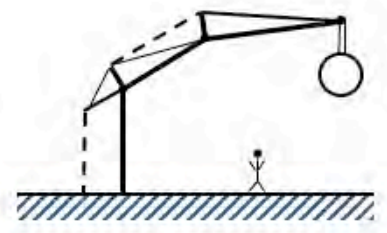

b. Hybrid Cable/Boom Manipulator.

Figure 2. Boom and hybrid Cable/Boom manipulators. are developed for each of the two types of manipulators considered. The equations take into account and allow major design inputs, including member lengths, boom reach, payload mass, planetary gravity value, boom material (density, strength and stiffness), actuator torque mass efficiency, maximum allowable tip deflection and factors of safety to be varied. In these derivations, the governing design condition such as, strength, stiffness, or stability is determined for each major sub-element of the manipulator and used to develop appropriate design relations. These design equations are applied to a wide range of loadings and sizes to provide an understanding of relative performance of different manipulators and to identify major design drivers. Attention is focused only on the manipulator concept with no consideration given to the platform on which the manipulator would be mounted and moved, end effectors that would be needed for various operations, or counter weights.

\section{Manipulator Systems Considered}

For earth based operations, wide use is made of the advantages offered by hydraulics. In vacuum operations such as would be found on the Lunar surface, hydraulics may not be feasible. In the current study, attention is restricted to two types of actuators. The first is a revolute joint powered by an electric motor with a very high gear reduction. This type of actuator is referred to herein as a geared actuator. The second consists of an electric winch actuator coupled to an offset lever arm to provide high local mechanical advantage and to reduce the loads in the members and the amount of gear reduction needed by the actuator. This second type of actuator is referred to herein as a cable actuator. This type of actuator is particular to the Hybrid Cable/Boom manipulator concept, extensively described in reference 1 and shown schematically in figure 2-b. For lightly loaded or short arms, a simple articulated and cantilevered boom driven by a geared actuator at the root can be used. However, for applications involving high loads or long reaches, offset cables provide a significant mechanical advantage that can reduce the mass and size of the lifting device.

The two major requirements for these devices for Lunar or planetary operations are to have low mass and package compactly for launch. For small manipulators a few meters in reach and with low force requirements, a simple device such as that shown in figure 2-a can be used. The boom manipulator shown in figure 2-a has a vertical member (kingpost) that can rotate about the azimuth at the base and has two geared actuators to drive the horizontal arm linkages for vertical motion, much like a conventional robotic arm. Simple folding of the arm provides compact packaging. Since this is basically a simple cantilever device, relatively large moments must be resisted by the arms and the geared actuators. For medium sizes, 5 to 10 meters in reach, a manipulator with cable actuators such as that shown in figure 2-b must be considered to control the growth of system mass as well as launch volume. Although the cable driven configuration, by including only pure tension and compression members, is more efficient, it can also be more complex (for equivalent number of degrees of freedom) and will require additional articulations of the offset members to accomplish compact packaging. To reduce the growth of manipulator mass, it may be necessary to use a truss for the compression members. If a truss is used, it will likely have a deployable cross-section to enable compact packaging for launch. Mass and component sizing relations are derived herein for the concepts shown in figures 2-a and 2-b. Example numerical results are presented for both concepts to demonstrate their relative performance for potential Lunar operations.

\section{Major Mass Performance Parameters}

In this section the major governing parameters of a cantilevered boom are established so that a quantitative mass comparison can be made between a wide variety of manipulator concepts, loads, and sizes. Since the design requirements for a lifting device will be a function of load and size, it is impossible to find one set of parameters that exactly covers all cases. For example, depending upon load and size, the sizing of the device may be limited by 
material strength, structural stiffness, or structural stability. In this section the major governing parameters are derived assuming material strength is the limiting design condition. In subsequent sections, the impact of stability and stiffness are included.

The reference manipulator considered for demonstrating the governing parameters is shown in figure 3-a. As shown in the figure, the manipulator has a maximum reach of distance $\mathrm{R}$ and is supported on a kingpost of height $\eta \mathrm{R}$. All tubular members are assumed to have a square cross-section with the dimensions shown in figure 3-b. The primary design load is a vertical tip force of magnitude $\mathrm{F}$. The major assumptions of this analysis are:

- The major design constraint is material strength,

- The tube wall thickness, $t$, is a prescribed minimum gage,

- The tubular walls are thin compared to the tube cross-sectional dimension,

- $\mathrm{a}$, is the average square tubular dimension as shown in figure $3-b$,

- The tube wall dimensions are not tapered along the length,

- The stress $\sigma_{\mathrm{T}}$ is selected as a relatively low value to account for knockdowns due to handling and joints,

- The mass of the actuators and joints are included with the factor, $\gamma$.

The thin tubular wall assumption leads to the following simplified relations for tube cross-sectional area, $\mathrm{A}_{\mathrm{T}}$, and moment of inertia, $\mathrm{I}_{\mathrm{T}}$ :

$$
A_{T}=4 a t
$$

And

$$
I_{T}=\frac{2}{3} a^{3} t
$$

Equations 1 and 2 enable a simple solution procedure and are adequate for preliminary design. If, a, is taken as the average tube cross-sectional dimension, the expression for area in equation 1 is exact. The error for moment of inertia in equation 2 is given by the following equation:

$$
\frac{I_{\text {exact }}}{I_{T}}=1+\frac{1}{(a / t)^{2}}
$$

For all designs considered in this paper, $\mathrm{a} / \mathrm{t}>10$, thus, the error in equation 2 is less than $1 \%$.

The maximum bending stress in the tube is at the root of the horizontal member and is given by

$$
\sigma_{T}=F S_{T} \frac{M C}{I_{T}}
$$

American Institute of Aeronautics and Astronautics 
where $\mathrm{C}=\mathrm{a} / 2$ and $\mathrm{M}=\mathrm{FR}$. Substituting for $\mathrm{I}_{\mathrm{T}}$ from equation 2 into equation 4 and solving for, a, yields:

$$
a=\left(F S_{T} \frac{3}{2} \frac{F R}{2 \sigma_{T} t}\right)^{1 / 2}
$$

The total mass, $\mathrm{M}$, of the manipulator is given by:

$$
M=A_{t}(R+\eta R) \rho_{T} \gamma=4 a t(R+\eta R) \rho_{T} \gamma
$$

Substituting for, a, from equation 5 into equation 6 and dividing by $\mathrm{R}$ yields the following mass merit function relation for the simple cantilevered manipulator:

$$
\frac{M}{R}=\rho_{t}(1+\eta) \gamma\left(F S_{T} \frac{12 t}{\sigma_{T}}\right)^{1 / 2}(F R)^{1 / 2}
$$

In equation 7, the major manipulator governing parameters are, $M / R$, and FR. To demonstrate the use of these parameters, they are plotted in figure 4 for the values assumed in Table 1.

The value for the constant, $\gamma$, which is a ratio of the mass of the tube, joints, and actuators, to the mass of the tubes was selected as 2.5 based on observed weights of several manipulator systems. This heuristic approach allows the establishment of a set of reference lines as presented in figure 4 against which alternate manipulator concepts can be compared. A low value of the allowable Table 1. Numerical values used to generate figure 4.

\begin{tabular}{|l|l|}
\hline Parameter & Value \\
\hline$\eta$ & 0.5 \\
\hline$\gamma$ & 2.5 \\
\hline $\mathrm{t}$ & 0.25 inches $(6.38 \mathrm{~mm})$ \\
\hline$\sigma_{\mathrm{T}}$ & $21,000 \mathrm{psi}(48.3 \mathrm{MPa})$ \\
\hline $\mathrm{FS}$ & 3 \\
\hline$\rho_{\mathrm{T}}$ & $0.3 \mathrm{lb} / \mathrm{in}^{3}\left(8302 \mathrm{~kg} / \mathrm{m}^{3}\right)$ for steel \\
\hline$\rho_{\mathrm{T}}$ & $0.1 \mathrm{lb} / \mathrm{in}^{3}\left(2767 \mathrm{~kg} / \mathrm{m}^{3}\right)$ for aluminum \\
\hline$\rho_{\mathrm{T}}$ & $0.06 \mathrm{lb} / \mathrm{in}^{3}\left(1660 \mathrm{~kg} / \mathrm{m}^{3}\right)$ for graphite epoxy \\
\hline
\end{tabular}
stress was chosen to allow for knockdowns due to cutouts, joints, and damage that may occur during operations. To establish these simplified mass reference lines, the allowable stress value was taken the same for all materials and only the density was changed. The value selected for the thickness, $6.38 \mathrm{~mm},(0.25$ ") was chosen to provide robustness against damage as well as to aid in keeping the tubular member cross-section size small to provide compact packaging. It is emphasized that this is not a design chart but is meant to provide a historical perspective for guiding future designs. As an example to demonstrate use of the chart, the Phoenix arm shown in Figure 4, which has aluminum links, lies very close to the aluminum line.

\section{Manipulator Mass Derivations}

In this section, the mass of three different manipulators will be derived; a boom manipulator, a

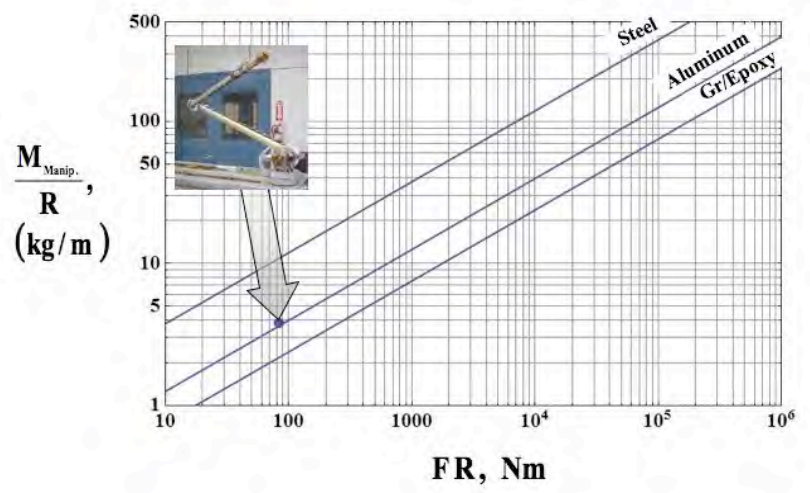

Figure 4. Mass merit function chart with Phoenix Arm. hybrid manipulator with tubular compression members, and a hybrid manipulator with truss compression members. 


\section{A. Boom Manipulator}

In the previous section a simplified cantilevered boom manipulator was used to derive the primary parameters for a manipulator mass index. In that derivation, the mass of the geared actuators were treated heuristically by assuming their mass was some percentage of the arm mass. In this section, a sizing algorithm is introduced for the geared actuators as well as the boom structure to provide a higher fidelity mass prediction model for manipulators. The structural study of the boom includes a strength, as well as a stiffness limited design.

To provide a sizing model for the geared actuators, it is assumed that the amount of torque, $\tau_{\mathrm{a}}$, a geared actuator can produce is proportional to its mass as

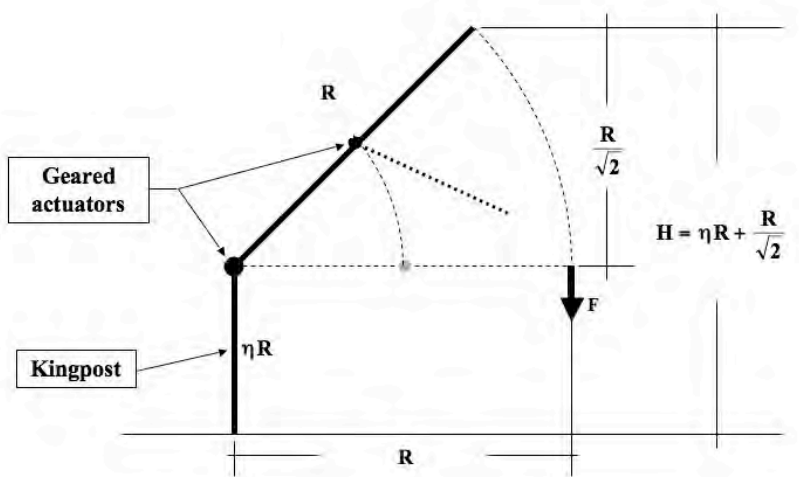

Figure 5. Assumed geometry and actuators for boom manipulator arm.

follows:

$$
\tau_{a}=K_{a} M_{a}
$$

Where $\tau_{\mathrm{a}}$ is the torque produced by the actuator, $\mathrm{K}_{\mathrm{a}}$ is the proportionality constant with units of $\mathrm{Nm} / \mathrm{kg}$, and $\mathrm{M}_{\mathrm{a}}$ is the mass of the actuator. For a required torque, equation 8 can be used to determine the actuator mass. The boom manipulator analyzed in this section, shown in figure 5, has two geared actuators as shown in the figure.

The derivation of the mass equation for the boom manipulator is similar to that conducted previously except that the mass of the geared actuators is determined from equation 8 rather than using the ratio $\gamma$. Thus, the manipulator mass is written as;

$$
M=4 a t(R+\eta R) \rho_{t} j+1.5 \frac{1}{K_{a}} F R
$$

Where $\mathrm{j}$ is a constant to account for joint mass of the tubes and FR is the torque $\mathrm{T}$ in the root actuator. The factor of 1.5 in the expression for the actuator mass takes into account that the actuator in the middle of the arm requires only one half the torque capability of the root actuator.

1. Strength Design

The expression for the cross-sectional dimension, a, of a manipulator designed for strength (see equation 5) is:

$$
a=\left(\frac{3}{2} \frac{F R}{2 \sigma_{T} t}\right)^{1 / 2}
$$

\section{Stiffness Design}

In this section a stiffness constraint is considered such that the deflection of the boom tip, $\Delta$, is limited to some small fraction of its length, $R$. The vertical deflection of tip of the manipulator as shown in figure 3 is written as:

$$
\Delta=\frac{F R^{3}}{3 E_{T} I}+\frac{F R(\eta R)}{E_{T} I} R=(1+3 \eta) \frac{F R^{3}}{3 E_{T} I}
$$


The first term is the tip beam deflection of the horizontal arm and the second term is the tip deflection due to beam rotation of the tip of the vertical member. Substituting I from equation 2 into equation 11 and solving for, a, yields:

$$
a=\left(\frac{1}{2}(1+3 \eta) \frac{F R}{E_{T} t \frac{\Delta}{R}}\right)^{1 / 3} R^{1 / 3}
$$

Substituting this value of, a, into equation 9 will yield the manipulator mass for a given stiffness constraint.

\section{B. Hybrid Manipulator with Tube Compression Members}

Numerical studies of the boom manipulator in the previous section have shown that size and mass of the required geared actuators become quite large with increasing loads. In an attempt to control the growth of the actuators, it is common to use cables with large offsets to provide a high mechanical advantage to reduce actuator torque requirements. A cable/boom manipulator that is considered in this paper is shown in figure 6. The manipulator has a maximum reach, $R$, and a kingpost height $\eta R$. It has two primary actuators, as shown in the figure, that drive the actuating cables (shown by dashed lines), so that actuating the cables changes the manipulator geometry. The cable offset from the support structure, $\mathrm{b}$, is assumed to maintain a constant ratio with respect to manipulator radius for any value of $\mathrm{R}$. This ratio, $\mathrm{b} / \mathrm{R}$, is the mechanical advantage that the cable/boom manipulator provides for reducing actuator torque. Holding $\mathrm{b} / \mathrm{R}$ constant controls the amount of torque that needs to be applied by the hoists for raising a payload mass at the tip. The parameter $\mathrm{c}_{\mathrm{b}}$ is a factor that accounts for the mass of the lower arm, (see Figure 6 and equation 33) to accommodate the required stiffness for system needs. Due to the offset cables, the torque requirements in the actuators are considerably lower that for the simple boom manipulator. The member loads resulting from a tip force $\mathrm{F}$, and an effective self mass, Me, are shown in figure 7 . The assumed effective mass, Me, acting at the mid-span of the horizontal member is approximated as:

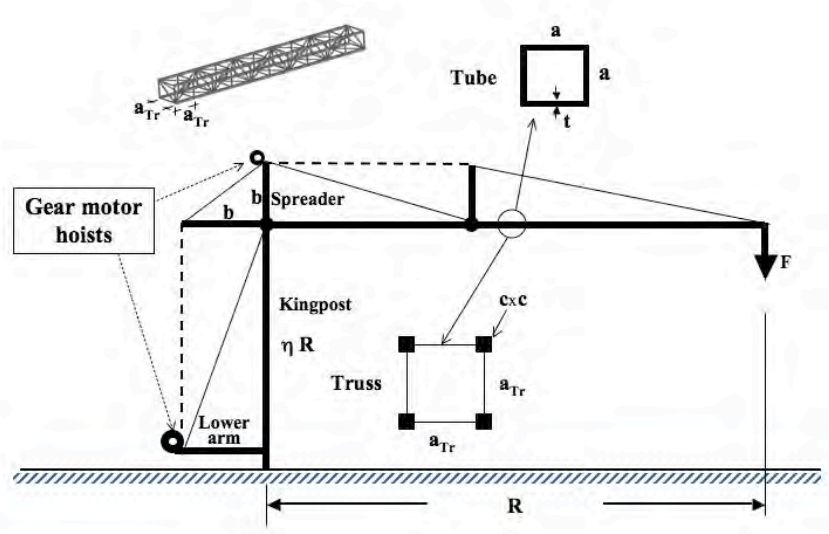

Figure 6. Assumed geometry and actuators for hybrid cable/boom manipulator with either square tubular, or square truss cross-section.

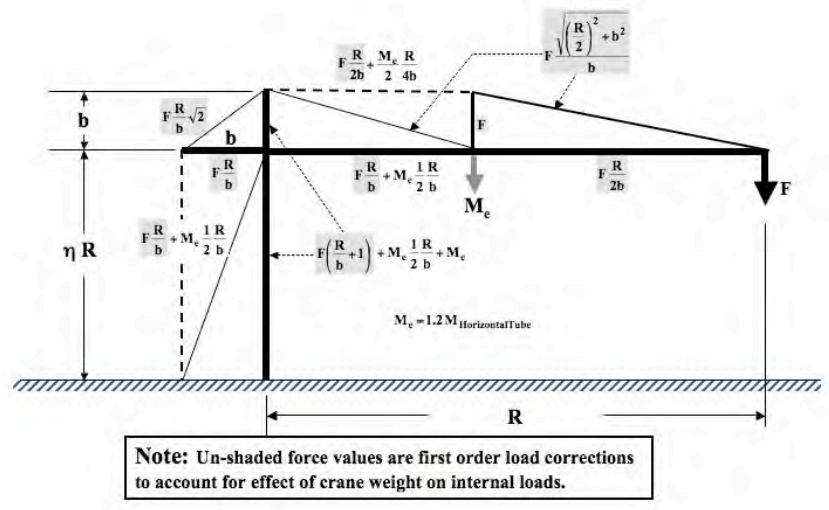

Figure 7. Member loads and dimensions for hybrid cable/boom manipulator.

$$
M_{e}=1.2\left(M_{\text {HorizontalBoom }}\right)
$$

The major component of the self mass is the mass of the boom arm of length R. Since it is difficult to include the effects of all self mass components such as the load alleviation from the mass on the left hand side of the vertical boom and the mass of a tip payload hoist, all other masses including the cables and rods are assumed to be included in the factor of 1.2 in equation 13. 


\section{Cable and Rod Diameters}

There are two primary actuation cables in the manipulator, shown by the dashed lines in figure 7. (All other tension members are assumed to be rods.) The forces in the cables, $F_{c}$, are also shown in the figure. In order to reduce the required hoist torque for lifting operations, a "block-and-tackle" arrangement of n cables could be used in place of a single cable. Such a system would reduce the cable force by $\mathrm{n}$ and thus reduce hoist torque required values. The required area of the cable can then be determined from the following equation:

$$
\sigma_{c}=\frac{F S_{c} F_{c}}{A_{c} n}
$$

where $\sigma_{c}$ is the ultimate stress of the cable, $A_{c}$ is the nominal area of the cable, $n$, is the number of cables, and FS $S_{c}$ is the factor of safety for the cable. The diameter of the cable is given by:

$$
d_{c}=\sqrt{\frac{4}{\pi} A_{c}}
$$

where $d_{c}$ is the maximum outside diameter of the cable. Using equations 14 and 15 and the cable loads from figure 7 , the diameters of the vertical cable, $\mathrm{d}_{\mathrm{cv}}$, and the horizontal cable, $\mathrm{d}_{\mathrm{ch}}$, are:

$$
\begin{gathered}
d_{c v}=\sqrt{\frac{4}{\pi} \frac{F S_{c}}{\sigma_{c}} \frac{1}{n}\left(F \frac{R}{b}+M_{e} \frac{1}{2} \frac{R}{b}\right)} \\
d_{c h}=\sqrt{\frac{4}{\pi} \frac{F S_{c}}{\sigma_{c}} \frac{1}{n}\left(\frac{1}{2} F \frac{R}{b}+\frac{M_{e}}{2}\left(\frac{R}{4 b}\right)\right)}
\end{gathered}
$$

Where $M_{e}$ is defined in figure 7. Similar to equation 17, the diameter of each of the solid rods (thin solid lines in figures 6 and 7) can be determined from:

$$
\begin{gathered}
d_{\text {rodh }}=\sqrt{\frac{4}{\pi} \frac{F S_{\text {rod }}}{\sigma_{\text {rod }}} F \frac{\sqrt{\left(\frac{R}{2}\right)^{2}+b^{2}}}{b}} \\
d_{\text {rodv }}=\frac{1}{\sqrt{2}} d_{\text {rodh }} \\
d_{\text {rodb }}=\sqrt{\frac{4}{\pi} \frac{F S_{r o d}}{\sigma_{\text {rod }}} F \frac{R}{b} \sqrt{2}}
\end{gathered}
$$


In these equations the subscripts, $\mathrm{h}$, and, $\mathrm{v}$, refer to the horizontal and vertical directions respectively, while $\mathrm{b}$ refers to the upper left rod in figure 6 between the two spreaders of length $b$.

4. Cable and Rod Masses

The masses of the cables and rods can be determined from:

$$
M_{c}=n \frac{\pi d_{c}^{2}}{4} L_{c} \rho_{c} j
$$

or,

$$
M_{r o d}=\frac{\pi d_{r o d}^{2}}{4} L_{r o d} \rho_{r o d} j
$$

Where $\mathrm{L}_{\mathrm{c}}$ or $\mathrm{L}_{\text {rod }}$ are the appropriate lengths of each cable or rod. The cable and rod lengths are multiplied by a factor of $\mathrm{j}$ to account for joint mass, such as end doubling and wrapping around the hoist drum or rod end fixtures.

5. Hoist Mass

The masses of the hoists are determined in the same fashion as was done for the boom actuators. For the cable driven manipulator, the hoist consists of a motor, gears, and a drum. The mass of the hoist, $\mathrm{M}_{\mathrm{H}}$, is determined from the following equation:

$$
\tau_{H}=K_{H} M_{H}
$$

In this equation, the required torque in the hoist, $\tau_{\mathrm{H}}$, is given by:

$$
\tau_{H}=(\text { Cable Load }) \frac{d_{H}}{2}
$$

Where $\mathrm{d}_{\mathrm{H}}$ is the diameter of the hoist drum. The minimum hoist diameter to cable diameter ratio is taken as 20 , based on bending restrictions given by Shapiro in reference 5, thus:

$$
d_{H}=20 d_{c}
$$

From equations 16,17 , and 25 , the diameters of the Drums, $d_{H}$, for the actuators that drive the vertical and horizontal cables are:

$$
\begin{aligned}
& d_{H v}=20 d_{c v} \\
& d_{H h}=20 d_{c h}
\end{aligned}
$$


The masses of the hoists, $\mathrm{M}_{\mathrm{H}}$, for the vertical and horizontal cables can then be determined from equations 23 , 24,26 , and 27 as:

$$
\begin{aligned}
M_{H v} & =\frac{1}{K_{H}} \frac{1}{n}\left(F \frac{R}{b}+M_{e} \frac{1}{2} \frac{R}{b}\right) \frac{20 d_{c v}}{2} \\
M_{H h} & =\frac{1}{K_{H}} \frac{1}{n}\left(F \frac{R}{2 b}+\frac{M_{e}}{2} \frac{R}{4 b}\right) \frac{20 d_{c h}}{2}
\end{aligned}
$$

\section{Size and Mass of Manipulator Tubes}

Since an assumption has been made that the tube thickness is a pre-selected constant $t_{\min }$, there is only one free design variable, a, for the tube. Thus, only a single constraint condition is needed. Numerical studies supported by finite element analyses indicated that the critical design condition for the cable/boom manipulator shown in figure 7 is Euler buckling of the vertical support post. The length of that member is $\eta \mathrm{R}+\mathrm{b}$ and is subjected to a compressive load as indicated in figure 7. The expression for the Euler buckling of the vertical support kingpost cantilevered from the bottom is written as:

$$
P_{E}=\frac{1}{F S} \frac{\pi^{2} E_{t} I}{4(\eta R+b)^{2}}
$$

The load in the vertical support kingpost from the tip load and self load is:

$$
P_{k p}=F\left(\frac{R}{b}+1\right)+M_{e} \frac{1}{2} \frac{R}{b}+M_{e}
$$

Using equations 2, 30, and 31, an expression for the cross-sectional dimension, a, can be found as:

$$
a=\left(\frac{3}{2 t} \frac{4 F S(\eta R+b)\left[F\left(\frac{R}{b}+1\right)+M_{e} \frac{1}{2} \frac{R}{b}+M_{e}\right]}{\pi^{2} E_{t}}\right)^{1 / 3}
$$

Although, a, was determined to prevent Euler buckling of the vertical king post, for sizing purposes, all tubes are assumed to be the same size.

7. Total Mass of Hybrid Cable-Boom Manipulator

The mass of the tubes and offsets, $\mathrm{M}_{\text {tubes }}$, is obtained by assuming that they all have the same cross-sectional section dimension, a, which is determined from equation 32 and is written as:

$$
M_{\text {tubes }}=4 a t\left(R+\eta R+3 b+c_{b} b\right) \rho_{t} j
$$


The mass of the two cables is written below, where the cable diameters are obtained from equations 16 and17:

$$
M_{\text {cables }}=\frac{\pi d_{c v}^{2}}{4}(j \eta R) \rho_{c}+\frac{\pi d_{c h}^{2}}{4}(j R) \rho_{c}
$$

The mass of the two hoists is determined from equations 28 , and 29 as:

$$
M_{\text {Hoists }}=L W H \frac{1}{K_{H}} \frac{1}{n}\left(F \frac{R}{b}+M_{e} \frac{1}{2} \frac{R}{b}\right) \frac{20 d_{c v}}{2}+L W H \frac{1}{K_{H}} \frac{1}{n}\left(F \frac{R}{2 b}+\frac{M_{e}}{2} \frac{R}{4 b}\right) \frac{20 d_{c h}}{2}
$$

where the factor LWH has been introduced into the hoist mass equation to account for a mechanism for level winding the cable, and for increases in torque that would occur because of drum diameter increase due to cable build-up.

The mass of the four rods is found from equations $18,19,20$ and 22 as:

$$
M_{r o d s}=\frac{\pi d_{r o d}^{2}}{4}\left(2 \sqrt{\left(\frac{R}{2}\right)^{2}+b^{2}}+\sqrt{(\eta R)^{2}+b^{2}}+\sqrt{2} b\right) \rho_{r o d} j
$$

The total mass of the hybrid cable/boom manipulator is now determined by adding the masses of all of the components as:

$$
M=M_{\text {tubes }}+M_{\text {cables }}+M_{\text {Hoists }}+M_{\text {rods }}
$$

\section{Hybrid Manipulator with Truss Compression Members}

The mass derivation of a cable/boom manipulator with solid square truss members is essentially the same as that for the cable/boom manipulator with square tubular support members as developed in the previous section. In fact, the only change that occurs in equation 37 is the expression for the mass of the tubes, $\mathrm{M}_{\text {tubes }}$, is replaced by an expression for the mass of the truss, $\mathrm{M}_{\text {truss }}$. The support tube is replaced by a truss with square bays as shown in figure 6 . The length of the longeron and batten members is $\mathrm{a}_{\mathrm{T}}$, and all members are assumed to have a solid square cross-section. The cross-sectional dimension of the longerons is, $\mathrm{c}$, while the cross-sectional area of the battens and diagonals are assumed to be $\beta$ times the area of the longerons, with $\beta$ taken as 0.5 in the present study. This reduced area accounts for the fact that the battens and diagonals have much lower loads than the longerons.

In the previous section for the tubular support structure, there was only one free design variable, a, since the tube thickness was assumed to be a constant. For the truss support structure, there are two free design variables, $\mathrm{a}_{\mathrm{Tr}}$, and c, thus, two constraints are required. The first is Euler buckling of the king post as in the previous section, and the second is Euler buckling of the longerons. The moment of inertia of the four longeron truss is:

$$
I_{T r}=a_{T r}^{2} c^{2}
$$

Thus, the constraint equation for Euler buckling of the kingpost is found from equations 30, 31, and 38 as: 


$$
\frac{1}{F S} \frac{\pi^{2} E_{t} a_{T r}{ }^{2} c^{2}}{4(\eta R+b)^{2}}=F\left(\frac{R}{b}+1\right)+M_{e} \frac{1}{2} \frac{R}{b}+M_{e}
$$

The moment of inertia of the longerons is:

$$
I_{\ell}=\frac{c^{4}}{12}
$$

And the longeron Euler buckling load is given by:

$$
P_{E \ell}=\frac{1}{F S} \frac{\pi^{2} E_{T r} I_{\ell}}{a_{T r}^{2}}
$$

Or from equations 40 and 41 :

$$
P_{E \ell}=\frac{1}{F S} \frac{\pi^{2} E_{T r} \frac{c^{4}}{12}}{a_{T r}^{2}}
$$

The constraint equation for longeron buckling is found from equations 31 and 42 as:

$$
4 \frac{1}{F S} \frac{\pi^{2} E_{T r} \frac{c^{4}}{12}}{a_{T r}{ }^{2}}=F\left(\frac{R}{b}+1\right)+M_{e} \frac{1}{2} \frac{R}{b}+M_{e}
$$

The solution of the two equations 39 and 43 for $\mathrm{a}_{\mathrm{Tr}}$ and c yields:

$$
\begin{gathered}
a_{T r}=\frac{2^{2 / 3} F S_{T r}^{1 / 6}\left(F\left(\frac{R}{b}+1\right)+M_{e} \frac{1}{2} \frac{R}{b}+M_{e}\right)^{1 / 6}\left(b^{2}+2 b R \eta+R^{2} \eta^{2}\right)^{1 / 3}}{3^{1 / 6} E_{T r}{ }^{1 / 6} \pi^{1 / 3}} \\
c=\frac{F S_{T r}{ }^{1 / 3}\left(F\left(\frac{R}{b}+1\right)+M_{e} \frac{1}{2} \frac{R}{b}+M_{e}\right)^{1 / 3}\left(b^{2}+2 b R \eta+R^{2} \eta^{2}\right)^{1 / 6}}{E_{T r}{ }^{1 / 3} \pi^{2 / 3}}
\end{gathered}
$$

The total mass of all of the truss members can now be found from the following equation: 


$$
M_{\text {Truss }}=4 c^{2}\left(R+\eta R+3 b+c_{b} b\right) \rho_{T r} j
$$

In this equation $4 \mathrm{c}^{2}$ is the area of the 4 longerons and the quantity inside the parentheses is the sum of the lengths of all the truss members. The last length in the parentheses, $b$, is multiplied by a factor $c_{b}$ to account for added bending stiffness that may be needed in the lower arm. An expression for the total mass of the truss cable/boom manipulator is given as;

$$
M=M_{\text {Truss }}+M_{\text {cables }}+M_{\text {Hoists }}+M_{\text {rods }}
$$

Where $M_{\text {Truss }}$ is obtained from equation 46 , the cable mass, $M_{\text {cables, }}$ from equation 34 , the hoist mass, $M_{\text {Hoists, from }}$ equation 35 , and the rod mass, $\mathrm{M}_{\mathrm{rods}}$, from equation 36 .

\section{Example Manipulator Sizing and Mass Results}

To provide insight into the performance of boom manipulators, tubular cable/boom manipulators, and truss cable/boom manipulators, the equations of the previous section were programmed in Mathematica and numerically exercised for a wide range of manipulator parameters. Except where noted, all calculations reflect nominal graphite/epoxy properties, and "g" was assumed to be $9.8 / 6 \mathrm{~m} / \mathrm{sec}^{2}$ so that all results are specific to the Lunar surface. The results of these studies are documented in the following sections.

\section{A. Assumed Values and Properties}

All results were obtained for the assumed values and properties listed in Table 2 unless otherwise noted. The cable offset from the support structure, $b$, is assumed to be the same percentage of the manipulator radius, $\mathrm{R}$, for all sizes. The ratio, $\mathrm{b} / \mathrm{R}$, is the mechanical advantage that the cable/boom manipulator provides for reducing actuator torque. Holding $\mathrm{b} / \mathrm{R}$ constant controls the amount of torque that needs to be applied by the hoists for raising the payload mass. The parameter $c_{b}$ which controls the lower arm stiffness was assumed as 2 for all cases. The modulus, $\mathrm{E}_{\mathrm{T}}$, of the tubes for both the boom manipulator and the cable/boom manipulator was taken as $12 \mathrm{msi}$. This modulus was selected as a readily achievable value for high strength graphite/epoxy with some angle plies for torsion and toughness. The modulus, $\mathrm{E}_{\mathrm{Tr}}$, for the truss longerons and rods was assumed to be $18 \mathrm{msi}$, reflecting that the truss longerons and the rods would be primarily unidirectional graphite/epoxy. The strength of the steel cable was taken as 104,000 psi. This value is representative of standard crane quality cables. The mass density of the steel cables, $\rho_{\mathrm{C}}$, was taken as $2 / 3$ of the density of steel to account for voids caused by the cable wire bundles. The densities of the rods, $\rho_{\mathrm{rod}}$, and the densities of the tubes and truss, $\rho_{\mathrm{T}}$, were taken as that of graphite epoxy. The thickness, $t$, of the tubes for both the boom manipulator and the cable/boom manipulator were assumed to be a constant equal to $0.25 \mathrm{in},(6.35 \mathrm{~mm})$. This assumption

Table 2. Assumed values used for examples.

\begin{tabular}{|l|l|}
\hline Parameter & Value \\
\hline $\mathrm{b}$ & $1.3 / 7.5 \mathrm{R}=0.173 \mathrm{R}$ \\
\hline $\mathrm{c}_{\mathrm{b}}$ & 2 \\
\hline $\mathrm{E}_{\mathrm{T}}$ & $12.0 \mathrm{msi}(82.7 \mathrm{GPa})$ \\
\hline $\mathrm{E}_{\mathrm{Tr}}$ & $18.0 \mathrm{msi}(124.0 \mathrm{GPa})$ \\
\hline$\sigma_{\mathrm{C}}$ & $104.0 \mathrm{ksi}(0.717 \mathrm{GPa})$ \\
\hline$\sigma_{\text {rod }}$ & $60.0 \mathrm{ksi}(0.414 \mathrm{GPa})$ \\
\hline$\rho_{\mathrm{C}}$ & $0.20 \mathrm{lb} / \mathrm{in}^{3}\left(5536 \mathrm{~kg} / \mathrm{m}^{3}\right)$ \\
\hline$\rho_{\text {rod }}$ & $0.06 \mathrm{lb} / \mathrm{in}^{3}\left(1660 \mathrm{~kg} / \mathrm{m}^{3}\right)$ \\
\hline$\rho_{\mathrm{T}}$ & $0.06 \mathrm{lb} / \mathrm{in}^{3}\left(1660 \mathrm{~kg} / \mathrm{m}^{3}\right)$ \\
\hline $\mathrm{t}$ & $0.25 \mathrm{inches}(6.38 \mathrm{~mm})$ \\
\hline $\mathrm{FS}$ & 5 \\
\hline $\mathrm{FS}$ & 3 \\
\hline $\mathrm{FS}$ & 3 \\
\hline $\mathrm{FS}$ & 3 \\
\hline$\eta$ & 0.5 \\
\hline $\mathrm{j}$ & 1.4 \\
\hline$\beta$ & 0.5 \\
\hline $\mathrm{K}_{\mathrm{H}}$ & $125 \mathrm{Nm} / \mathrm{kg}$ \\
\hline $\mathrm{K}_{\mathrm{A}}$ & $125 \mathrm{Nm} / \mathrm{kg}$ \\
\hline $\mathrm{N}$ & $4 \mathrm{or} 1$ \\
\hline$\Delta / \mathrm{R}$ & 0.02 \\
\hline $\mathrm{M}_{\mathrm{e}}$ & 0.0 \\
\hline & \\
\hline & \\
\hline
\end{tabular}


was made to ensure handling toughness of the tubes. The factor of safety for the cables, $\mathrm{FS}_{\mathrm{C}}$, was taken as 5 , a value recommended by Shapiro for cranes. The factors of safety for all other members were taken as 3 . The allowable stress in the graphite/epoxy rods was assumed to be a low value of 60,000 psi to account for impact damage. The height of the king post was assumed to be one half of the manipulator radius resulting in $\eta=0.5$. A factor, $j$, to account for joint mass in the tubes and trusses was taken as 1.4. In the current numerical studies the hoist torque/mass factor $\mathrm{K}_{\mathrm{H}}$ was assumed to be $125 \mathrm{Nm} / \mathrm{kg}$. In a subsequent section this value is varied to demonstrate its effect on manipulator mass. Except where noted, the number of cables, $n$, is taken as 4 . The value of the deflection constraint, $\Delta / \mathrm{R}$, for the boom manipulator was taken to provide manipulator tip deflections similar to that experienced by the cable/boom manipulator. Because studies included here have shown that the mass of Lunar manipulators is $5 \%$ or less of the payload, the self weight factor, Me, is neglected for that case. For Mars or Earth applications the effect of Me would be greater due to the higher gravitational values.

\section{B. Sizing and Mass Versus Tip Force}

In this example, a plot of manipulator mass as a function of applied tip force, F, is presented. Since these masses are in terms of applied force, the results are independent of the specific gravitational field. Thus, these results are applicable to Mars as well Lunar applications within the constraint of assuming $\mathrm{Me}=0.0$. As can be seen in figure 8 , the actuators represent a significant portion of the boom mass, while the mass of the hoists for the cable/boom manipulators is low. The mass of the hoists for the tubular cable/boom and the truss cable/boom manipulators is the same. The difference in the masses of these two manipulators is due to the difference in the masses of the tubular and the truss support structure. The mass of the hoists is small due partly to a "block and tackle" arrangement of 4 cables being used in the calculations for figure 8 .

\section{Sizing and Mass Versus Reach Radius}

In this example, figure 9 shows a plot of manipulator mass as a function of radius. Results are presented for a load level of $12,000 \mathrm{~N}$, representing a heavy lift application, such as a habitat module. Since these masses are in terms of applied force, the results are independent of the specific gravitational field. Thus, these results are valid for Mars as well Lunar applications.

\section{Sizing and Mass Versus Actuator Efficiency}

In this example, a plot of manipulator mass as a function of $\mathrm{K}_{\mathrm{H}}$, is presented. (Note that in all cases, $\mathrm{K}_{\mathrm{A}}=\mathrm{K}_{\mathrm{H}}$.) Since these masses are in terms of applied force, the results are independent of the specific gravitational field. The primary purpose of these results is to demonstrate the sensitivity of manipulator mass to the

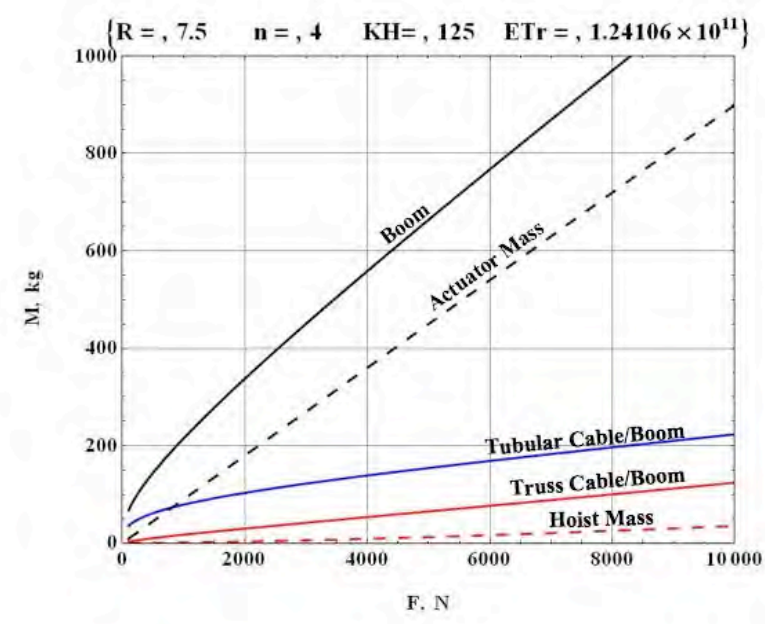

Figure 8. Manipulator mass as a function of applied tip force, $F$.

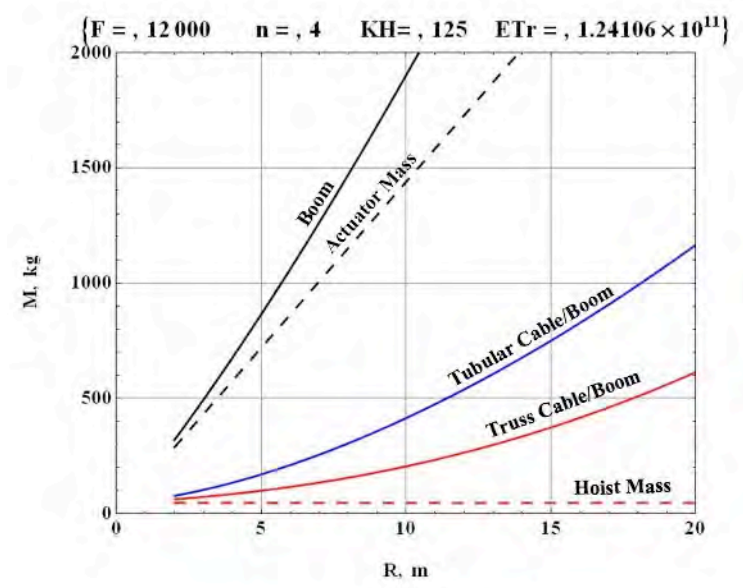

Figure 9. Manipulator mass as a function of radius, R. actuator torque mass performance parameter $\mathrm{K}_{\mathrm{A}}$. For a typical set of design conditions, figure 10 shows that the boom manipulator is highly sensitive to the assumed value of actuator performance, in contrast to the hybrid cableboom manipulator, which is not. A plot of the ratio of the truss cable/boom mass to the boom manipulator mass is presented in figure 11 for two cases of radius and load levels. The curves bound all combinations of radius and load between the cases shown. For low efficiency actuators, (low values of KA $100 \mathrm{Nm} / \mathrm{kg}$ ), it can be seen that the truss cable/boom manipulators have masses that are on the order of $10 \%$ of boom manipulators while for higher efficiency actuators, $(\mathrm{KA} \sim 500 \mathrm{Nm} / \mathrm{kg}$ ), the hybrid cable/boom manipulators still only have $20 \%$ of the mass of a corresponding boom manipulator. 


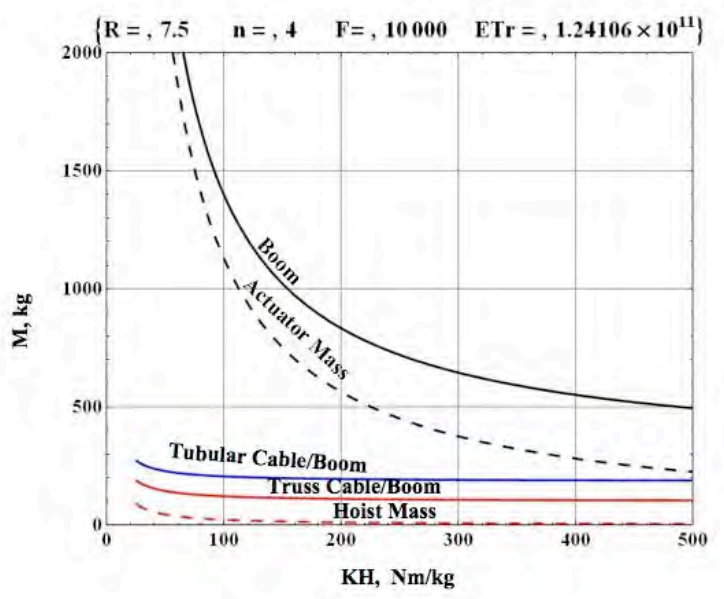

Figure 10. Manipulator mass as a function of actuator torque/mass performance, KA.

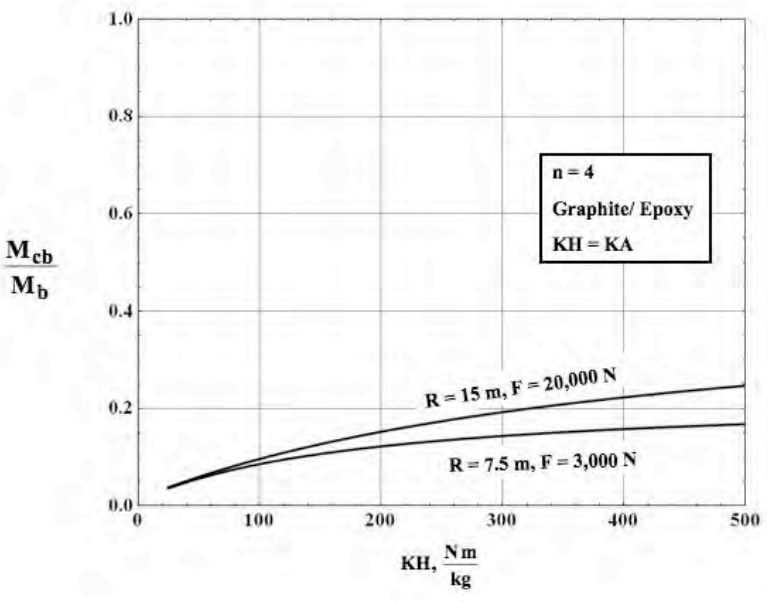

Figure 11. Comparison of hybrid truss cable/boom manipulator mass to boom manipulator mass.

\section{E. Sizing and Mass Versus Planetary Surface Gravity}

In this example a study was made to determine the percentage of manipulator mass with respect to the payload mass for various levels of gravity, g. In particular, results were obtained for values of $g$ for Lunar, Mars, and Earth applications for two different values of manipulator radius, $7.5 \mathrm{~m}$, and $15 \mathrm{~m}$. Results of the study, presented in figure 12 , show that the mass ratio varies linearly for different values of radius. From the figure it can be concluded that the mass ratio decreases with decreasing values of $g$. For zero $g$ as with the Shuttle Remote Manipulator System (SRMS), the mass ratio is extremely small. For Lunar applications, the mass ratio is only about $2 \%$ for $\mathrm{R}=$ $7.5 \mathrm{~m}$, and about $5 \%$ for $\mathrm{R}=15 \mathrm{~m}$.

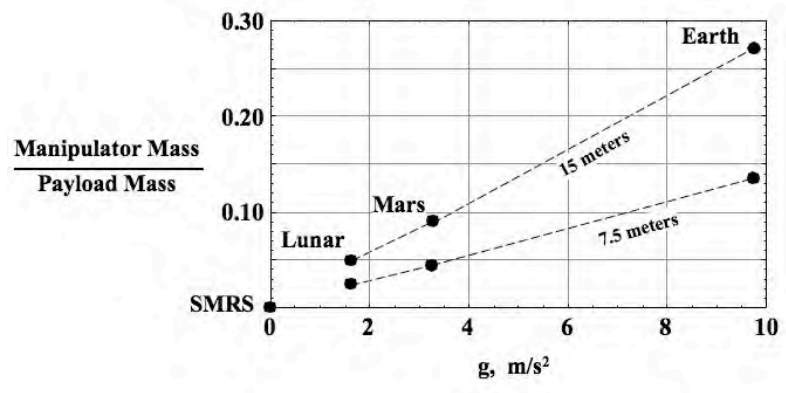

Figure 12. Ratio of hybrid truss cable/boom manipulator mass to payload mass as a function of $\mathrm{g}$.

\section{F. Performance Summary}

In this example, a performance parameter chart comparing various manipulators is developed and presented in figure 13. The parameters on the chart were derived previously in the paper and the curves for the $\mathrm{Gr} / \mathrm{Ep}$, Aluminum, and Steel boom manipulators are the same as presented in figure 4. The two lower mass curves for $\mathrm{R}=7.5 \mathrm{~m}$ and $\mathrm{R}=15 \mathrm{~m}$ on figure 13 are for hybrid truss, cable/boom manipulators for $n=4$. Although the two mass curves were developed as a function of tip force, F, so that they would be generally applicable, the end points of each curve are identified in terms of Lunar mass as examples. The end point mass values were chosen to bound expected applications for Lunar operations. For Mars or Earth applications, the appropriate value of gravity would need to be used. Although the simple performance parameters developed in this paper do not completely eliminate the effect of length from the two lower manipulator curves, the

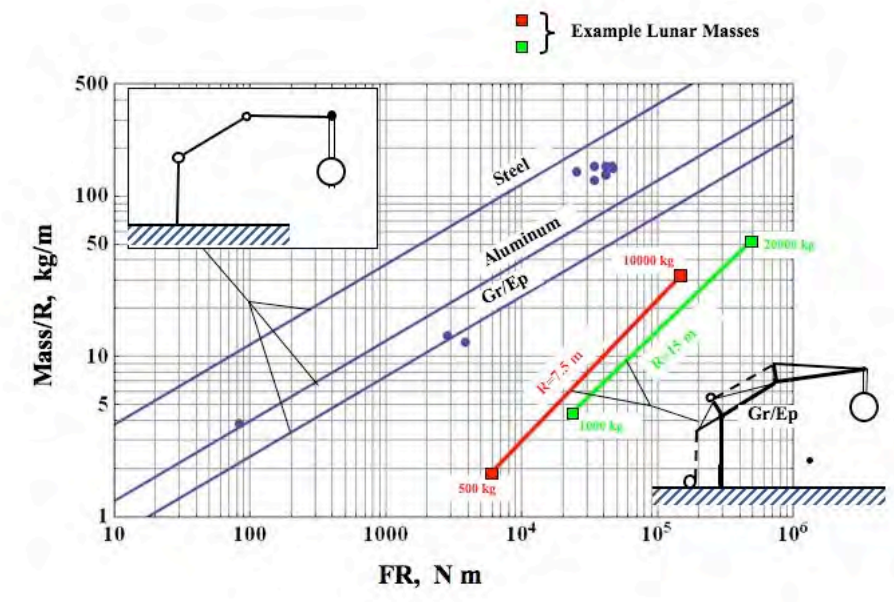

Figure 13. Performance parameter plot comparing various manipulator concepts.

American Institute of Aeronautics and Astronautics 
results provide information for manipulator mass estimation over a wide range of operational sizes and loads. These two curves bound all masses for the lengths and load ranges presented.

\section{G. Impact of Materials on Mass}

In this example, the masses of several aluminum and graphite/epoxy (gr/ep) manipulators, normalized with respect to the mass of a graphite/epoxy truss cable boom manipulator, are compared in figure 14. The number of cables, n, was equal to 4 for all cases in this example. For the aluminum manipulator designs, the following properties are substituted for modulus and density: $\mathrm{E}_{\mathrm{T}}=\mathrm{E}_{\mathrm{Tr}}=68.9109 \mathrm{~Pa}(10$ $\mathrm{msi})$, and $\rho_{\mathrm{T}}=2767 \mathrm{~kg} / \mathrm{m}^{3}$.

For very high values of load, the gr/ep tubular truss cable/boom manipulator is on the order of twice the mass of the gr/ep truss cable/boom manipulator, and the gr/ep boom manipulator is on the order of six to eight times the mass of the gr/ep truss cable/boom manipulator. For low values of load, the mass of the gr/ep truss cable/boom manipulator is significantly lower than that of both the gr/ep tubular cable/boom manipulator and the gr/ep boom manipulator. As is the case for other compression designed structures,

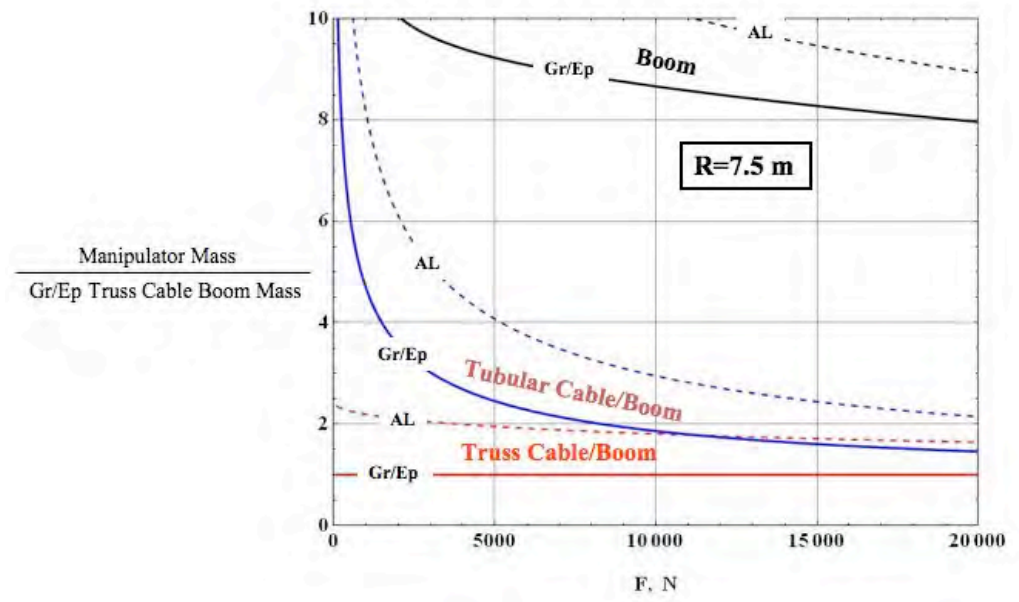

Figure 14. Various manipulator masses compared to that of a graphite/epoxy hybrid truss cable/boom manipulator.

trusses are very efficient for low loadings because the dimension of the open truss cross-section can grow to provide high bending stiffness at a low cost in mass. At very high values of loads, the truss mass approaches that of the tubular cross-section. The mass savings for the gr/ep truss and tubular cable/boom manipulator are about $50 \%$ compared with aluminum, while the savings is $20 \%$ or less for the boom structure. This reduction in percentage savings for the boom manipulator is primarily because the total mass has such a high percentage of actuator mass.

\section{Concluding Remarks}

The mass and sizing characteristics of manipulators for Lunar (with extension to Mars) planetary surface applications were investigated by analyzing three structural configurations: a simple cantilevered boom with a square tubular cross-section; a cable/boom configuration with a square tubular cross-section support structure; and a cable/boom configuration with a square truss cross-section support structure. Design procedures, including the details of the actuator mass, were developed for the three configurations and numerical examples were given.

A new set of performance parameters have been developed that relate the mass of manipulators to a loading parameter. These parameters enable the masses of different manipulator configurations to be compared over a wide range of design loads and reach envelopes (radii). The use of these parameters was demonstrated in the form of a structural efficiency chart using the newly considered manipulator configurations as well as several previously developed systems.

To understand the performance of Lunar manipulators, the design procedures were exercised on the three manipulator configurations assuming graphite/epoxy materials for the tubes and trusses. It is also assumed that the actuators are electric motor, gear reduction systems. Numerical results for manipulator masses and sizes were presented for lengths of $7.5 \mathrm{~m}$ and $15 \mathrm{~m}$ over a range of payload masses from $500 \mathrm{~kg}$ to $20,000 \mathrm{~kg}$. Results were also presented that demonstrate the sensitivity of manipulator mass to operational radius, tip force, and actuator efficiency. The effect of the value of gravitational force on the ratio of manipulator-mass to payload-mass was also shown. Finally, results were presented to demonstrate the relative mass reduction gained by using graphite/epoxy compared to aluminum for the support structure. Specific conclusions from this study are as follows:

Simple cantilevered boom manipulators are attractive (for their simplicity) at small sizes, ( 2 to $5 \mathrm{~m}$ ), and low load capability, $(20$ to $100 \mathrm{~N}$ ). This is exemplified by the Mars Phoenix robotic arm that is $2.3 \mathrm{~m}$ long, has a tip force capability of $36 \mathrm{~N}$, and has a mass of $8.2 \mathrm{~kg}$. In a Lunar gravitational field, this arm could handle $22 \mathrm{~kg}$, giving a manipulator mass to payload mass ratio of 0.37 . Although this mass ratio is acceptable for very small 
systems, the ratio is prohibitive for manipulators that would be required to handle large masses, $(500 \mathrm{~kg}$ to 20,000 $\mathrm{kg}$ ), in Lunar or Mars outpost operations.

The mass of the torque actuators required for simple cantilevered boom manipulators can represent 70 to $80 \%$ of the total manipulator mass, depending upon actuator efficiency and loading levels. In this study, attention was focused on manipulator concepts that significantly reduce actuator requirements.

A cable/boom manipulator configuration provides three major improvements in efficiency as compared with the cantilevered boom manipulator. First, the offset of the cable provides a deep structure that has much higher efficiency than the boom manipulator. Second, the offset cable provides a high mechanical advantage that reduces required actuator torque levels. Third, the cable configuration allows the use of a "block-and-tackle" arrangement that provides additional mechanical advantage for reducing actuator torque levels. These three improvements were shown to reduce the manipulator mass by a factor of five or more.

For the cable/boom system, a manipulator with a truss support structure is about $50 \%$ of the mass of a cable/boom manipulator with a square tubular structure and about $15 \%$ of the mass of a cantilevered boom manipulator.

A truss support structure is more efficient primarily due to the fact that its open cross-section provides a higher bending stiffness with less mass. Although this larger cross-section provides a more efficient bending member, it could result in a packaging volume penalty. To improve this situation, it would be desirable to have a truss structure whose cross-section could collapse for compact packaging.

The use of graphite epoxy for truss cable/booms provides about a 50\% mass savings as compared to aluminum. This mass saving is a result of a higher assumed modulus, (18 msi for gr/ep and $10 \mathrm{msi}$ for aluminum), and lower density for the graphite epoxy.

This study showed that manipulator mass reduces dramatically with a reduction in gravitational value. For Lunar operations the percentage of cable/boom manipulator mass to payload mass was $2-5 \%$, compared with $15-$ $30 \%$ for Earth operations. This study assumed the same level of advanced technologies for all $\mathrm{g}$ levels. The implication of these results is that the mass of manipulators for Lunar applications is quite low.

\section{References}

1. Dogget, W.; Dorsey, J.; Collins, T.; King, B.; and Mikulas, M.: A Versatile Lifting Device for Lunar Payload Handling, Inspection and Regolith Transport Operations. Proceedings of Space Technology and Applications International Forum - STAIF 2008. AIP Conference Proceedings Volume 969, Editor Mohamed S. El Genk, American Institute of Physics, 2008

2. Mikulas, M.; and Yang, L.; Conceptual Design of a Multiple Cable Crane for Planetary Surface Operations. NASA TM104041, Jaunary 1991.

3. Taylor, R.; Mikulas, M.; and Hedgepeth, J.: A Two Cable, Six Link Boom Crane for Lunar Construction. AIAA 93-1340.

4. Taylor, R.; Mikulas, M.; and Hedgepeth, J.: A Linearized, Discrete Radius Pulley Model for Finite Element Analysis. AIAA 94-1645.

5. Shapiro, H.; et. al.: Cranes and Derricks. McGraw-Hill, Inc., 1980, $2^{\text {nd }}$ Edition, 1991.

6. Mikulas, M.; Collins, T.; Doggett, W.; Dorsey, J.; and Watson, J.: Truss Performance and Packaging Metrics. Proceedings of Space Technology and Applications International Forum (STAIF-2006). AIP Conference Proceedings Volume 813, Editor Mohamed S. El-Genk, American Institute of Physics, Melville, New York, 2006. 